MATEC Web of Conferences 22,01045 (2015)

DOI: $10.1051 /$ matecconf/ 20152201045

(C) Owned by the authors, published by EDP Sciences, 2015

\title{
Design of Underground Current Detection Nodes Based on ZigBee
}

\author{
Deyu Wei \& Hucheng Wu \\ Henan College of Industry \& Information Technology, Jiaozuo, Henan, China
}

\begin{abstract}
At present, most current detection devices of underground power equipment in coal mines of China are equipped with the cable monitoring network. Certain problems such as difficult circuit extension and maintenance exist there. With the help of ZigBee technology, it is able to monitor the underground current of monitoring regions in coal mines safely and effectively. Major advantages include extremely low system cost, safe data transmission, flexible networking and ultra-large network capacity.
\end{abstract}

Keywords: ZigBee technology; current detection; sensor child node; router child node; coordinator node

\section{INTRODUCTION}

Most current detection devices of underground power equipment in coal mines of China are now equipped with the cable monitoring network. Poor underground working conditions would cause difficulties in the installation and maintenance of the cable monitoring system as well as in the circuit extension with tunneling.

ZigBee technology is mainly used to construct the wireless personal area network with a low bit rate. Members of the network are called node devices. The acquisition of all kinds of information in monitoring regions can be realized by installing a large number of sensor nodes. Major advantages include extremely low system cost, safe data transmission, flexible networking and ultra-large network capacity.

\section{CURRENT MONITORING SYSTEM}

\subsection{ZigBee technology}

The shape of "Zigzag" formed in the process of honey-gathering shares such information as direction, distance and position of food sources. Bees are small in figure with less energy consumption and lower efficiency of information transmission. People use ZigBee technology to represent the wireless communication technology with low cost, small volume, small energy consumption and low transmission rate. It becomes the first choice of the wireless current monitoring system due to the merits mentioned earlier. There are mainly three topological structures of the ZigBee wireless network, namely tree shape, star shape and net shape.

\subsection{System operating principle}

Through the current transformer, the monitoring system transforms the primary side current into the secondary side current and then transforms the secondary side current into digital signals, which are transmitted to the receiving device through the wireless transmission module. The receiving device conducts the real-time monitoring on digital signals transmitted from the wireless module. When digital signals are transmitted, these data will be stored in the buffer memory and then transmitted to the upper computer through a wired or a wireless module. The upper computer is able to conduct real-time monitoring, receiving and analysis on information transmitted from the receiving device. Information is preserved in corresponding tables of the database in real time so that it can be displayed in forms of table and diagram. Besides, the current value transmitted by a certain or some acquisition devices in a certain stage can be retrieved and extracted, transformed, and displayed. The system will send out warning information in time when a certain value exceeds the predetermined threshold.

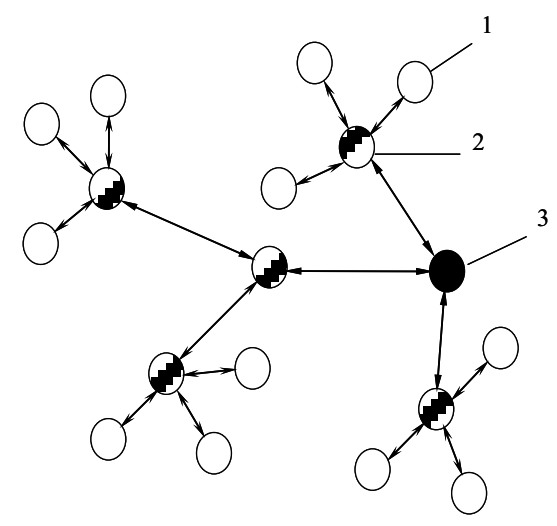

Figure 1. System architecture

1-Sensor child nodes; 2-Router child nodes; 3-Coordinator nodes 


\section{MATEC Web of Conferences}

\subsection{System architecture}

The terrain of the coal face is complicated and a great deal of data needs to be collected. According to the underground environmental characteristics of the coal mine, it is suggested to adopt the tree-cluster topological structure to construct the ZigBee network. The entire network includes sensor child nodes, router child nodes and coordinator nodes. The underground roadways of the coal mine are relatively long whereas the transmitting power of the sensor child nodes is small, so the router child nodes can be used to realize the multiple-hop transmission of frames.

The coordinator is the center of the whole network, which joins router nodes and collected nodes into the network constructed by the coordinator with network addresses so that they can communicate with the upper computer and upload data to the management center in real time. Router child nodes have the function of packets transmission. It establishes a channel for the communications transponder between the coordinator and the sensor child nodes for the convenience of information transmission. Sensor child nodes are responsible for the acquisition of current signals and router child nodes accomplish the reporting of data information. The system architecture is shown in Figure 1.

\section{HARDWARE DESIGN OF NODES}

\subsection{Sensor child nodes}

(1) Model selection of the current sensor

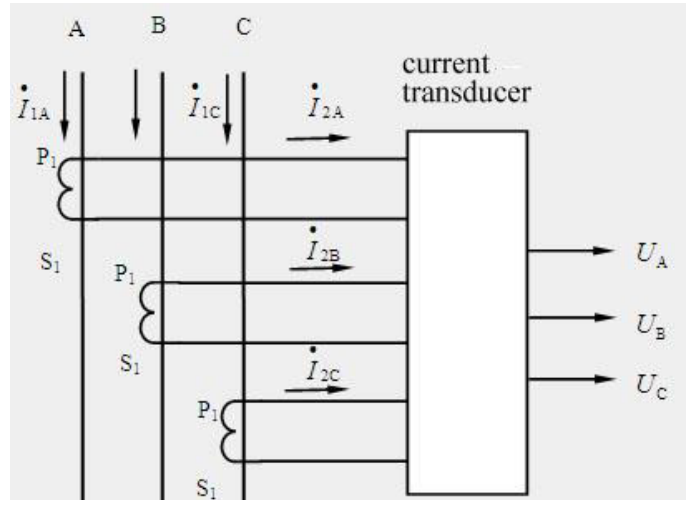

Figure 2. The schematic diagram of the current sensor

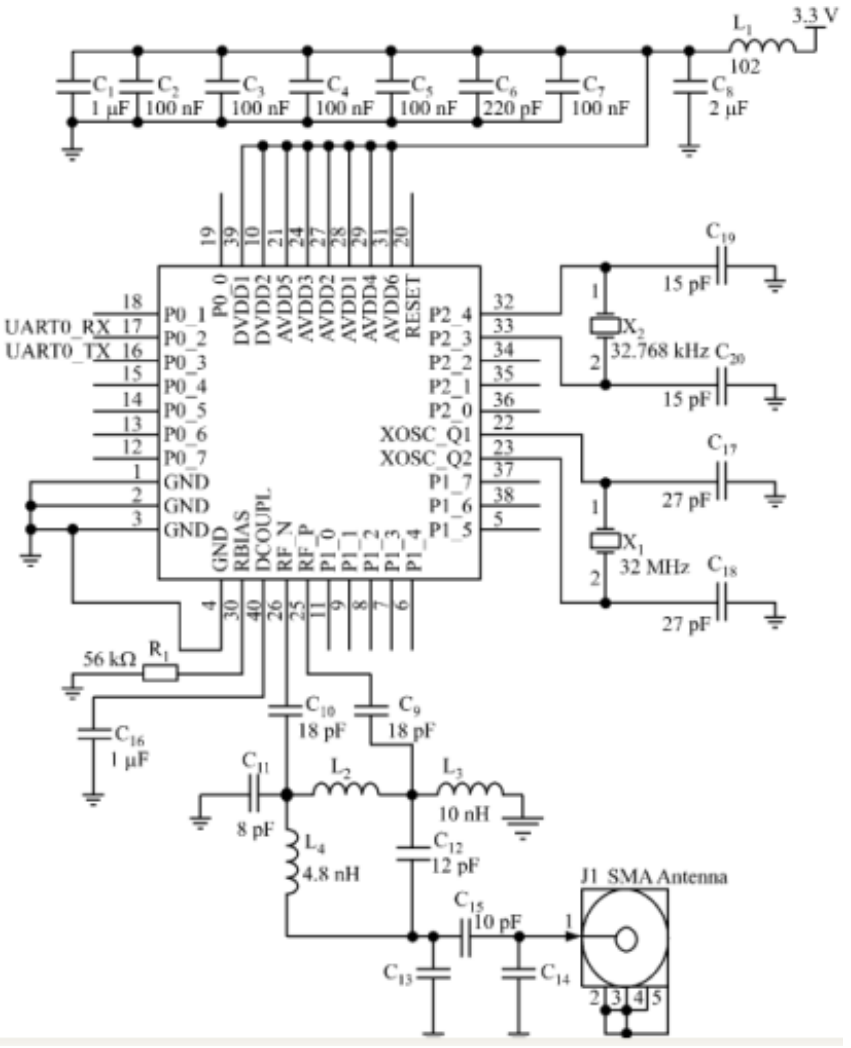

Figure 3. The circuit diagram of sensor child nodes 
Strong current signals cannot be collected and processed by the single-chip microcomputer. It is necessary to first transform the primary current into the smaller secondary current through the current transformer and then transform the smaller secondary current into voltage signals that can be received and handled through the current transducer. The process is shown in Figure 2.

According to the actual testing needs, the JIC-C21-600P1O2 alternating current transducer is adopted. JIC-C21-600P1O2 is a measuring module that transforms the measured current into DC voltage signals output proportionally to the primary side current in line with the electromagnetic induction principle. The primary side and the secondary side are highly insulated. It features high precision, high linearity, high integration density, small volume, simple structure, long-term stability of operation and the adaptability in various working environments. The rated input current is $600 \mathrm{~A}$, the rated output voltage is $0-5 \mathrm{~V}$, and the rated operating voltage of the current transducer is $24 \mathrm{~V}$.

\section{(2) CC2530 chip}

CC2530 is a new generation of SoC chip developed by the IT Company based on the ZigBee/IEE802.15.4 standard. With an $8 \mathrm{kB}$ RAM and a $256 \mathrm{kB}$ flash memory, it integrates a high-powered RF transceiver and an optimized low-consumption 8051 microprocessor, including an 8-channel 7-12 bits ADC, 2 USART and 21 general $\mathrm{I} / \mathrm{O}$ ports.

CC2530 is a wireless transmitting chip with certain control functions. It is able to complete all functions required by this design without the need for additional control chips. So, CC2530 is able to realize the transmission and receiving of wireless signals.

(3) The overall circuit design of the physical acquisition device

The P0.0-P0.7 port on the CC2530 chip is an ADC channel, which converts collected analog signals into digital signals without the need of other auxiliary circuits. As shown in Figure 3, $U_{A}, U_{B}$ and $U_{C}$ output by the current transducer are respectively connected to the P0.7 port, the P0.6 port and the P 0.5 port.

\subsection{Router child nodes}

Being affected by various underground environmental factors, the reliable transmitting distance of sensor child nodes is $100 \mathrm{~m}$. In order to transmit the sensor's information, router child nodes should be added to play the role of a transfer station, which forwards the data of sensor child nodes to coordinator nodes and enlarges the distance of data transmission. Router child nodes are generally installed in the middle position or the cornering of a long roadway. This is determined by the physical length of the roadway. A CC2591 radio frequency front-end is added at the front end of CC2530 to improve the transmitting power and the receiving sensitivity. The interface circuit of CC2530 and CC2591 is presented in Figure 4.

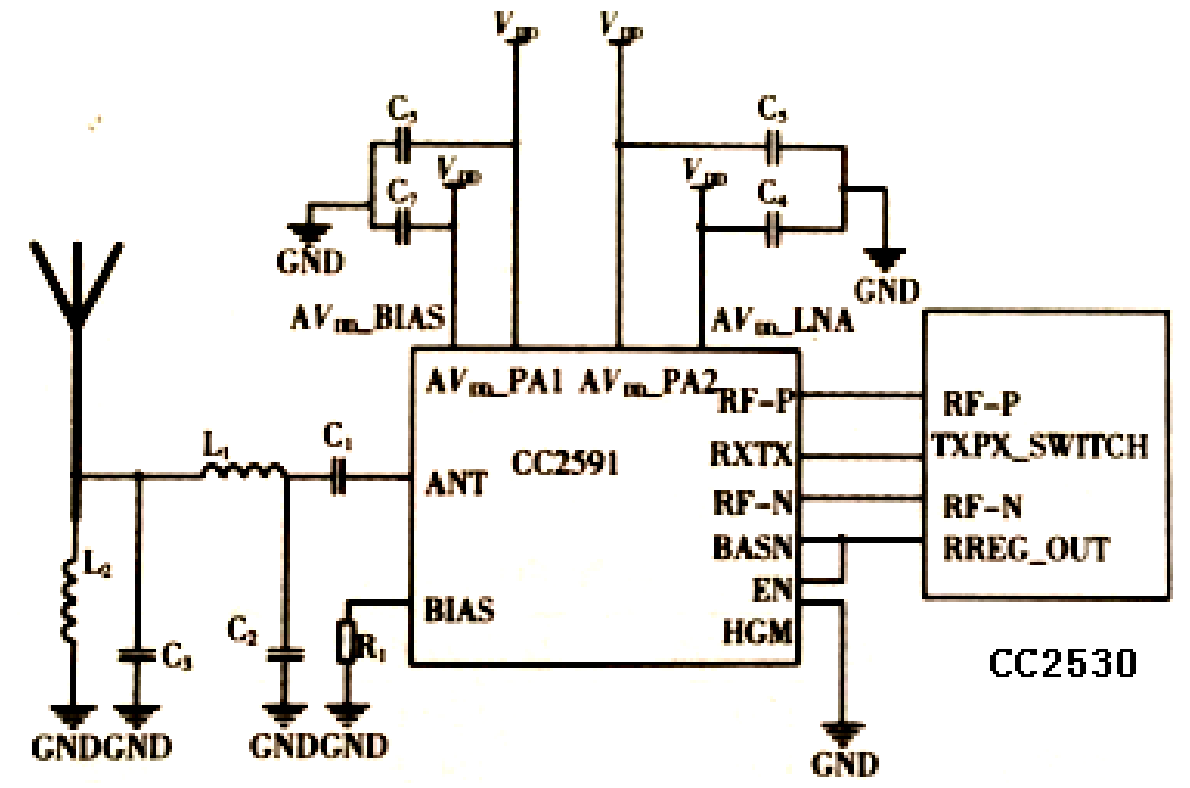

Figure 4. The interface circuit of CC2530 and CC2591 


\section{MATEC Web of Conferences}

\subsection{Coordinator nodes}

The coordinator is responsible for networking and communications, which uploads the collected information to the management center. It is the center of the entire network, mainly including the CAN bus interface, the liquid crystal display and the alarm indication circuit. As the center of the whole network, it must guarantee the long-term operation without power failure. The external power source if normally adopted for power supply. The system structure is shown in Figure 5.

\section{SOFTWARE PROCESS DESIGN}

Initialize the hardware platform after the electrification of coordinator nodes. Execute the operating system and start the ZigBee network. Upload received information to the upper computer in the monitoring state. Sensor child nodes are different from router child nodes in the operating mode but basically the same with router child nodes in the procedure. CC2530 has four kinds of power dissipation modes. The current consumption in the sleep state is only 0.9 $\mu \mathrm{A}$. In order to reduce the power dissipation, sensor child nodes can be designed not only in the mode of sleep-work-sleep but also in the sleep mode when they are off operation. The flow charts of the coordinator and sensor child nodes are shown in Figure 6

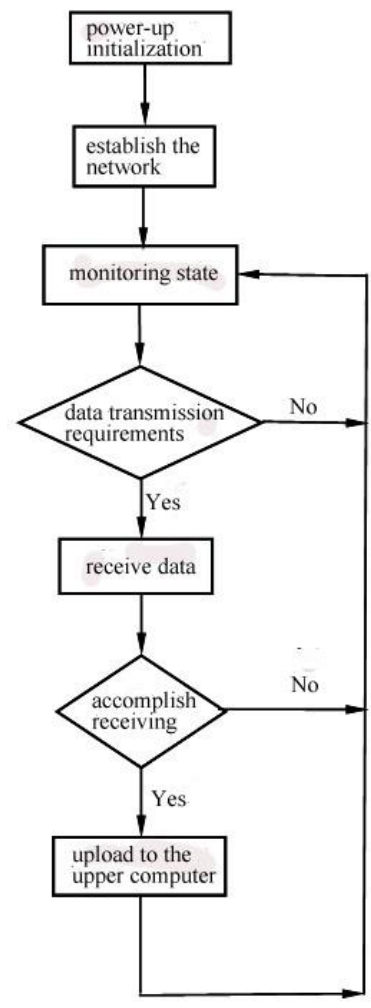

AIR7.60 is selected as the design and development environment. AIR7.60 is the application layer software programmed on the basis of Z-STACK-2.4.0-1.4.0 protocol stack.

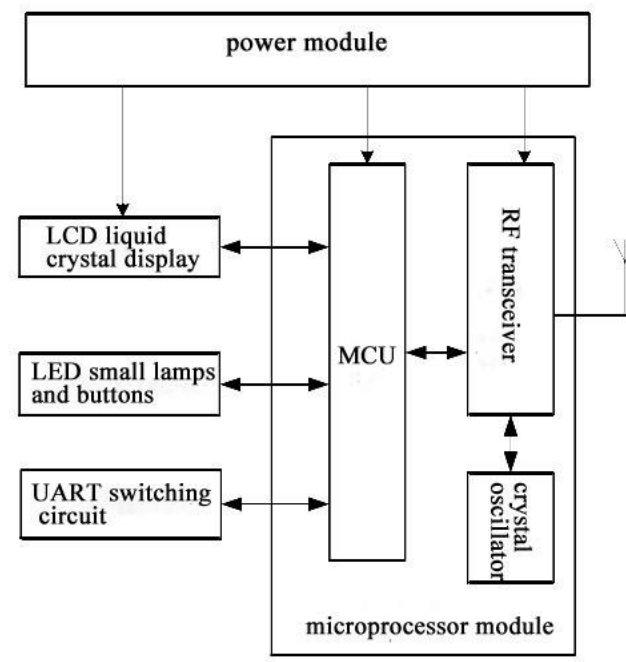

Figure 5. The structure chart of coordinator nodes

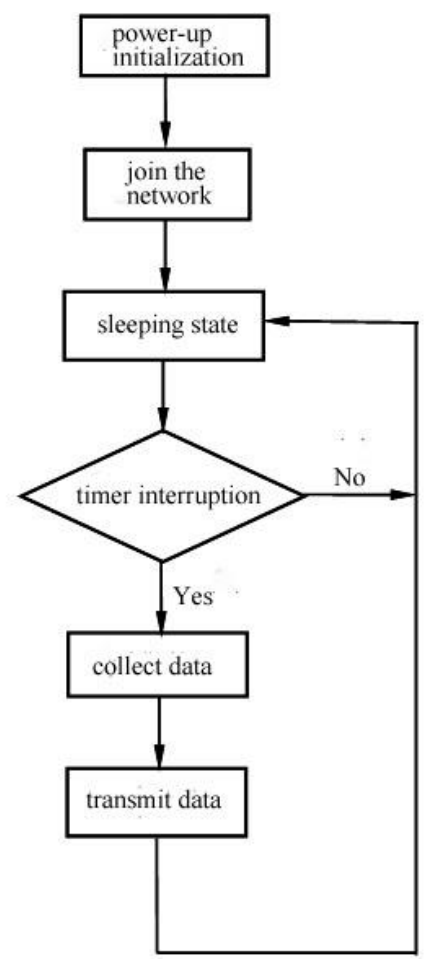

Figure 6. The flow charts of the coordinator and sensor child nodes 
ICETA 2015

Table 1. Estimated current and measured current.

\begin{tabular}{|l|l|l|l|l|l|l|l|}
\hline \multicolumn{9}{|c|}{ Fully mechanized coal mining face } \\
\hline No. & Device names & Number & Type & $\begin{array}{l}\text { Rated power } \\
\mathrm{kW}\end{array}$ & $\begin{array}{l}\text { Rated voltage } \\
\mathrm{kV}\end{array}$ & $\begin{array}{l}\text { Estimated } \\
\text { current A }\end{array}$ & $\begin{array}{l}\text { Measured } \\
\text { current A }\end{array}$ \\
\hline 1 & Coal cutter & 1 & $\begin{array}{l}\text { MG200/500- WD } \\
\text { K })\end{array}$ & 500 & 1140 & 300.1 \\
\hline 2 & $\begin{array}{l}\text { Flectional scraper } \\
\text { conveyer }\end{array}$ & 1 & SGZ730/400 & $2 \times 200$ & $660 / 1140$ & 120.5 \\
\hline 3 & $\begin{array}{l}\text { Reversed loader } \\
\text { Extensible belt con- } \\
\text { veyor }\end{array}$ & 1 & SZZ730/160 & 160 & $660 / 1140$ & 99.1 & 99 \\
\hline
\end{tabular}

\section{PERFORMANCE TEST}

The system is tested on electromechanical devices of the fully mechanized coal mining face of a certain coal mine. The working face has such devices as a coal cutter, a flectional scraper conveyer, a reversed loader and an extensible belt conveyor. Measured values are the data measured by special instruments. Data results indicate that the current error of the system is smaller than or equal to $0.1 \mathrm{~A}$. The difference between the estimated value and the measured value is mainly the error of the sensor itself, which satisfies the operating requirement, which is shown in Table 1.

\section{CONCLUSION}

Many shortcomings of the cable network can be overcome by ZigBee technology in the monitoring of the underground current. Sensor child nodes have such merits as small volume, low power dissipation and being convenient for the underground installation. Tree-cluster network increases the network coverage degree. It has a good promotional value and application prospects.

\section{REFERENCES}

[1] Zhang, D., Li, C.L. \& Xu, Y., et al. 2010. Design of the coal mines safety monitoring system based on ZigBee technology, Safety in Coal Mines, 41(7): 77 -80.

[2] Yang, J. 2011. A study on the safety monitoring system of low concentration coal gas pipelines, Automation \& Instrumentation, 155(3): 28-31.

[3] Peng, F., Wang, J.Q. \& Shi, C.H. 2013. Design and implementation of the refrigeration house monitoring system based on ZigBee, Journal of Chinese Agricultural Mechanization, 34(9): 248-250.

[4] Li, X.Z., Hu, X.Z. \& Feng, X.H., et al. 2014. Design of the coal mine roadway pressure monitoring system based on ZigBee, Industry and Mine Automation, 40(4): 19-22.

[5] Zhu, Y.K., Li, Z.B. \& Jiang, Y.Y., et al. 2014. The underground electric network leakage protection system of the wireless sensor network based on ZigBee, Industry and Mine Automation, 40(5): 15-17.
[6] Bo, Y.Q., Ouyang, M.S. \& Li, Y.L., et al. 2014. The hydrological information monitoring system of coal mines based on ZigBee, Industry and Mine Automation, 40(10): 84-87. 